\title{
INFANT'S GROWTH THROUGHT THE FIRST YEAR OF LIFE AND MATERNAL PRE- PREGNANCY BODY MASS INDEX
}

\author{
E. Verduci, G. Radaelli, E. Salvatici, C. Paramitiotti, E. Riva, G. Banderali \\ Department of Pediatrics, San Paolo Hospital University of Milan, Milan, Italy
}

\begin{abstract}
Aim: To assess whether an association may exist between maternal pre-pregnancy Body Mass Index (BMI) and infant's growth throughout the first year of life.

Methods: Prospective observational cohort study including 1246 at term, singleton, healthy newborns. Mothers were interviewed before discharge about pre-pregnancy weight, sociodemographic data, lifestyle and were given a diary to record the type of feeding during the study period. Maternal pre-pregnacy BMI was categorized according to the World Health Organization criteria as: underweight (BMI $<18.5 \mathrm{~kg} / \mathrm{m}^{2}$ ), normal-weight $\left(18.5 \mathrm{~kg} / \mathrm{m}^{2}<\mathrm{BMI}<25 \mathrm{~kg} / \mathrm{m}^{2}\right)$ and overweight (BMI $\geq 25 \mathrm{~kg} / \mathrm{m}^{2}$ ). Infant's growth (weight, length, BMI) data were collected during visits scheduled at 1, 3, 6, 9, 12 months of life, and z-scores were calculated.
\end{abstract}

Results: Birth weight was lower in infants born to underweight (mean $3289 \mathrm{~g}$, SD 384) than normal-weight (mean 3416 g, SD 408) and overweight mothers (mean 3426 g, SD 431) $(\mathrm{P}<0.05)$. Throughout the first year of life, the infant's BMI z-score ranged between 0.37 and 0.77 in the whole sample but was significantly higher in infants born to overweight than underweight and normal-weight mothers from 6 months onwards (maximum $\mathrm{P}=0.024)$. Significance of difference decreased $(\mathrm{P}=0.072)$ after adjusting for confounders (breastfeeding, solid foods introduction and type, mother age,educational level. No overall significant difference occurred among underweight, normal-weight and overweight mothers for length z-score.

Conclusions: The infant's BMI z-score pattern throughout the first year of life may be associated with maternal pre-pregnancy BMI. 